\title{
A Marine GIS-based Alert System to Prevent Vessels Collision with Offshore Platforms
}

\author{
Sheng-Long Kao \\ National Taiwan Ocean University, Taiwan, slkao@mail.ntou.edu.tw \\ Ki-Yin Chang \\ National Taiwan Ocean University, Taiwan \\ Tai-Wen Hsu \\ National Taiwan Ocean University, Taiwan
}

Follow this and additional works at: https://jmstt.ntou.edu.tw/journal

Part of the Fresh Water Studies Commons, Marine Biology Commons, Ocean Engineering Commons, Oceanography Commons, and the Other Oceanography and Atmospheric Sciences and Meteorology Commons

\section{Recommended Citation}

Kao, Sheng-Long; Chang, Ki-Yin; and Hsu, Tai-Wen (2021) "A Marine GIS-based Alert System to Prevent Vessels Collision with Offshore Platforms," Journal of Marine Science and Technology. Vol. 29: Iss. 5, Article 1.

DOI: $10.51400 / 2709-6998.2462$

Available at: https://jmstt.ntou.edu.tw/journal/vol29/iss5/1

This Research Article is brought to you for free and open access by Journal of Marine Science and Technology. It has been accepted for inclusion in Journal of Marine Science and Technology by an authorized editor of Journal of Marine Science and Technology. 


\title{
A Marine Gis-based Alert System to Prevent Vessels Collision with Offshore Platforms
}

\author{
Sheng-Long Kao ${ }^{a, b}$, Ki-Yin Chang ${ }^{a, b}, *$, Tai-Wen Hsu ${ }^{a, b}$ \\ ${ }^{a}$ Intelligent Maritime Research Center, National Taiwan Ocean University, Taiwan \\ ${ }^{\mathrm{b}}$ Center of Excellence for the Ocean Engineering, National Taiwan Ocean University, Taiwan
}

\begin{abstract}
How to effectively use an intelligent aid-to-navigation (AtoN) to prevent ships from accidently colliding with offshore marine platforms (OMPs) is an important issue for maritime environment. This study proposes a novel collision alert system with three components to process Automatic Identification System (AIS) data in the cloud and broadcast AtoNs to ships in order to avoid collision with OMPs. The first component receives as input fuzzy linguistic variables (vessel size, relative speed, and approach distance) from the AIS data and calculates the radius of the guarding ring. The second component serves a Marine GIS (MGIS), where the system simulates the alert based on a fuzzy guarding ring at one of three levels: green (continuous light), amber (slow flash and alarm), and red (flash and fast alarm). The third component is a warning indicator device, signaling the risk of collision between moving ships and offshore platforms. The MGIS component can also be used on an OMP to alert approaching ships and to warn offshore platform workers and vessel traffic service (VTS) on-duty operators. The ability of the proposed system to protect OMPs from vessel collisions has been verified. Other potential applications include offshore wind turbines, oil rigs, or other working platforms.
\end{abstract}

Keywords: Virtual AtoN, Marine platform, Automatic identification system (AIS), Marine geographic information system (marine GIS)

\section{Introduction}

$\mathrm{T}$ raditional aid-to-navigation (AtoN) facilities include lighthouses, lightships, beacons, buoys, waterways, traffic separation schemes (TSS), shore navigational aids, and other warning facilities used to enhance safety by providing more opportunities to obtain lines of position [7]. Based on their navigational purposes, virtual AtoNs can be divided in two types: (1) maritime assisted navigation and (2) maritime hazardous obstacle facilities. These AtoNs improve navigation safety by displaying the positions of hazardous obstacles. Hazardous maritime facilities include offshore wind turbines, work platforms (such as oil rig platforms, gas drilling platforms, and subsea maintenance platforms), and drifting ships. Hazardous obstacle warning facilities are needed because ships and offshore work platforms may be unaware of a dangerous situation and be at risk of collision due to a lack of sound/light alarm devices for collision avoidance alerts. Those dealing with hazardous obstacle facilities (such as the newest wreck, not marked on the Electric Nautical Chart) must rely on confirmation from electronic navigation instrumentation and the crew's clear judgment. An AtoN is the most important navigational guide when navigating at night. In fact, most ship incidents are caused by unfamiliarity with the local navigation environment and navigational aids. Ship collisions or shipwrecks are often caused by merchant navigators' lack of attention to hazardous obstacle facilities.

In recent years, offshore wind turbines have been located in deeper waters and also in the vicinities of traffic lanes. Therefore, ship collision represents a major concern [2]. However, several vessel accidents 
have occurred as a result of the auto-pilot causing a collision with an offshore wind turbine [4]. Many researchers have studied collision risk and assessed the consequences of collisions between sea vessels and offshore wind farms $[1,10,13,15,16]$. The occurrence of such incidents can be substantially decreased by proposed virtual navigation aids reliant on a GIS-based system. Some researchers also used AIS data or developed the idea of employing multipliers to study the Safety Assessment Model for Shipping and Offshore on the North Sea (SAMSON model) $[9,14]$.

In this study, the collision alert distance of marine platforms depends on sea and ship conditions. In the case of fog or rough water, visibility can be drastically reduced, and a collision event may occur [2]. The safety distance for collision alerts should be increased due to visibility being decreased. Therefore, this study refers to the well-known fuzzy logical theory of Zadeh [20] and Buckley [3]. In previous work, three fuzzy membership functions used the length and velocities of merchant ships, as well as sea conditions, to establish the radius of the guarding circle $R_{r e} *(1+D)$, where $R_{r e}$ is the safety radius for the ship domain, as defined by Goodwin [5], and is 0.9 nautical miles (N.M). D is the output value by fuzzy inference for a fuzzy guarding ring of merchant ships [8]. The guarding radius will be displayed on the MGIS platform. In this research, fuzzy logic theory is applied to collision alert distances and situations by three linguistic variables, vessel size, relative speed, and approach distance, to provide the first component of marine GIS.

In the application device, AIS Class B data were used to establish a fuzzy virtual navigation-aided system to provide warnings for collision avoidance and safety between moving ships, offshore platforms, and obstruction facilities. The AIS data were used to establish a fuzzy virtual AtoN system to provide warning signals for collision alerts when ships travel along a coastline near hazardous obstacle facilities. If an offshore marine platform is at risk of ship collision, it is necessary to propose a safety plan with a collision avoidance warning to avoid an accident. In this first component of GISbased alert systems, the dynamic and static data from AIS transceivers are processed and calculated by an FLC to obtain the collision risk index and then converted into a look-up table to provide nearby ships and marine platforms with a warning alert. For vessels equipped with AIS, a shore station receives AIS data processed by MGIS. The alert level index is transmitted in virtual AtoN form [19] to the vessel traffic service (VTS) or the ship's Electronic Chart Display and Information System (ECDIS) for collision avoidance. Automatic warnings about navigational aids and hazard avoidance facilities with the proposed acousto-optic flash warning mechanism were shown to enhance maritime traffic safety. This proposed new warning indicator mode based on a GIS includes three levels of risk, green (continuous), amber (slow flash and slow alarm), and red (fast flash and treble alarm) lights, to warn moving ships and offshore platforms. According to experimental results, relevant information based on a GIS can facilitate virtual aids to navigation and automatic alerts for collision avoidance.

\section{AIS/VAtoN/MGIS integrated system}

Recently, the IALA (International Association of Lighthouse Authorities) has extended the concept of AIS in developing Virtual Aids to Navigation (VAtoN). IALA defines a Virtual Aid to Navigation as follows: "A virtual aid to navigation does not physically exist but is a digital information object promulgated by an authorized service provider that can be presented on navigational systems" [19]. Various types of AtoN are provided by IALA and can be utilized by flag state authorities according to the need for actual AtoN units to transmit data to relevant shore stations: (1) a real (physical) AtoN is a special AIS transponder physically installed on an AtoN; (2) a synthetic AIS AtoN is implemented in cases where AtoN equipment cannot be installed with an AIS unit; and (3) a virtual AtoN, as defined by IALA guideline 1081 on Virtual Aids to Navigation, does not physically exist but is instead a digital information object promulgated by an authorized service provider that can be presented on navigational systems. Simply stated, the IALA draft guidelines on Virtual Aids to Navigation show that a virtual AtoN is a virtual navigational aid that normally communicates by VHF in an AIS message $[6,18]$. Navigation-related symbols have been approved and adopted by IMO. It can be displayed on ECDIS or radar displays that support AIS message type 21 [7].

Geographic information can be assembled into meaningful data through the analysis functions and simulation processes provided by the software. The resulting data can be used for planning by policymakers [11,12]. It is possible to apply the concept of geographic information systems to the analysis and expression of relevant information concerning the oceans. This study combined fuzzy logic control theory (FLC) and MGIS for ship collision alerts to develop a Spatial Decision Support System (SDSS) at sea. This is referred to as the Fuzzy Marine Geographic Information Systems (FMGIS). 
The FMGIS created by this study employs the tools of collection, management, analysis, simulation, expression, fuzzy algorithm, and prediction to integrate a variety of relevant spatial information. The various marine databases and tools are applied to simplify the appearance of the marine world. After the information is collected and treated, it is analyzed and can then assist in spatial decisionmaking for collision alerts.

In this research simulation, the site marked by the cross symbol "+" is the AIS transmitting shore base station, and the green-right and red-left virtual AtoNs (buoys) are shown in Fig. 1. These virtual AtoNs represent the TSS by VTS broadcast. In the application device, a shore base station (VTS with marine GIS) is combined with the MGIS and AIS. This station will receive all information from vessels installed with shipborne AIS and simultaneously broadcast AIS message 21 to the surveillance area. All these vessels are able to acknowledge the virtual AtoN symbols. Based on this mechanism, these virtual symbols can represent the marine platforms on each vessel's ECDIS. The configuration of a virtual AtoN system is shown in Fig. 2.

In the future, the AIS and MGIS could be combined to provide land VTS via an AIS broadcasting system that uses virtual AIS. Therefore, virtual maritime traffic control lights, waterways, and aids to navigation will be displayable on a ship's nautical electronic chart and VTS stations. When this system builds up virtual aids to navigation, hazardous obstacle facilities, and virtual radio navigation aids through virtual AIS, it will be able to replace the physical navigational aid facilities. Because virtual AISs have fewer carbon emissions, low maintenance costs, and real-time scheduling capabilities, the system will not need to install any navigational aids, and will show the AIS information on the ECDIS at VTS.

\section{Model of fuzzy collision alert system}

The proposed alert system for the AIS/VAtoN/ MGIS is based on the concept of a danger index and fuzzy guarding radius calculated by the first component of the GIS. The calculated danger (alert) index $[0,10]$, described as the level of danger criterion, enables the GIS-based alert system to make a proper decision for collision alerts [8]. In the proposed method, the size of the guarding radius is determined by the fuzzy logic method and the danger index for vessel collision is cloud processing for the first component. The details of these concepts are described below.

\subsection{Model of fuzzy guarding radius for three alert stages}

The first priority of a navigational ship is collision avoidance at sea, but weather conditions are difficult to predict and rough seas and other bad weather conditions can make relative speed, sea state, visibility, ship maneuvering, or relative distance

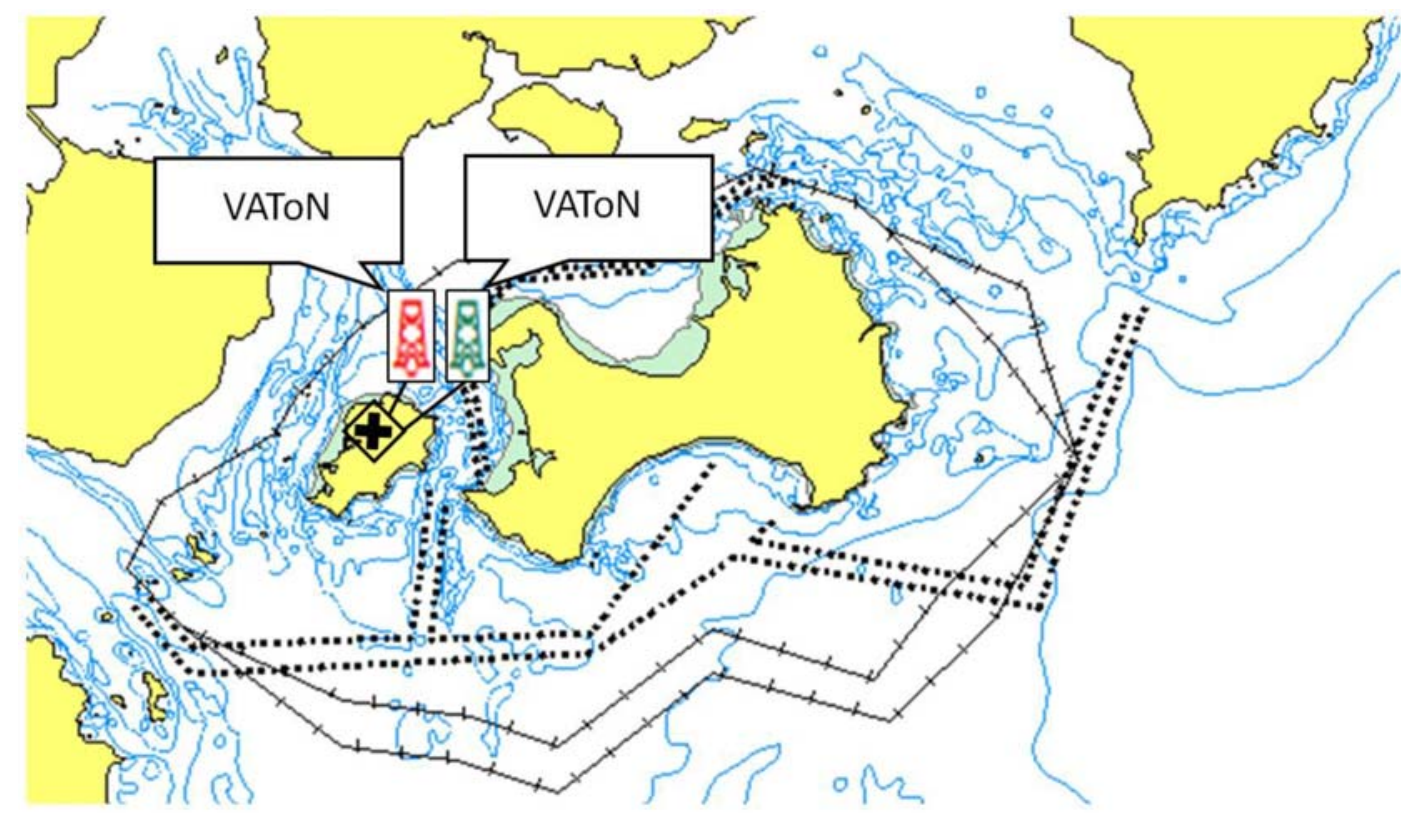

Fig. 1. Virtual buoys (AtoNs) presented on MGIS. 


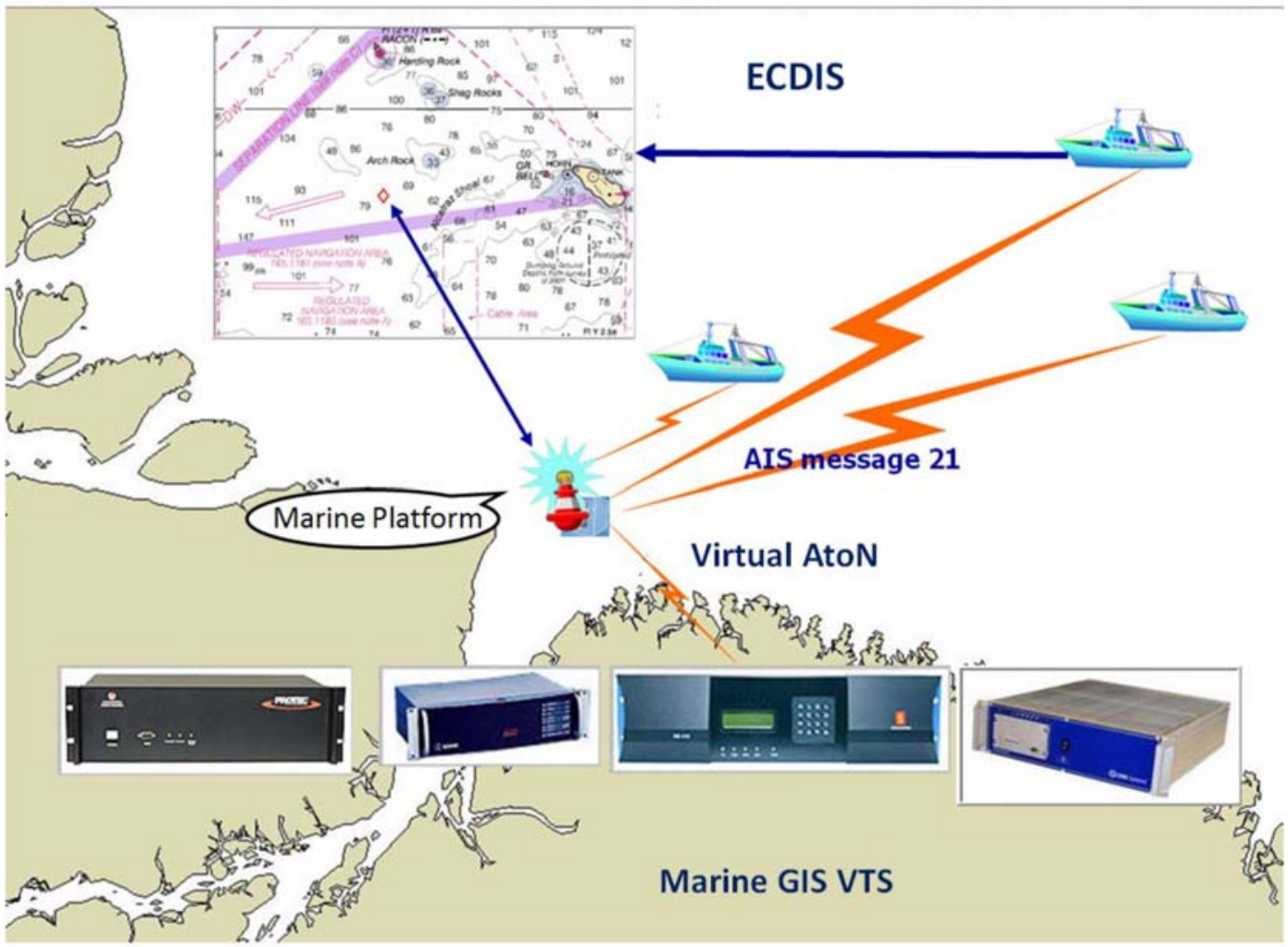

Fig. 2. Virtual AtoN system configuration.

become "ambiguous" for different captains. Therefore, fuzzy logic is very suitable for OMP collision alert systems. The FLC method and fuzzy rules are used to determine the different guard circle radii. In bad weather with poor visibility and rough seas, the Fuzzy Guarding Radius (FGR)/Ship Domain (SD) will be larger [17]. In good weather with high visibility and a calm sea, the FGR/SD radius will be smaller [8].

Fuzzy logic control can be used based on given input values, and many influences can be eliminated. These influences can arise from human perceptions or uncertainties due to environmental conditions. A fuzzy system can contain four principal elements, including fuzzification, a fuzzy rule base, a fuzzy inference engine, and defuzzification.

In order to solve most real-world fuzzy phenomena, fuzzy theory was developed from the foundation of fuzzy sets. It is mainly used to express fuzzy concepts that cannot be precisely defined, particularly when there is a better expression to describe the peculiar fuzzy characteristics of human language. The focal point of fuzzy theory is a mathematical model for a fuzzy concept that cannot be defined by humans. The characteristics of fuzzy things treated by humans, which describe human experience and knowledge, are then drawn in order to be used by computers [20]. Generally, fuzzy logic is employed to design a fuzzy control system through the concept of fuzzy theory. The basic structure of a fuzzy control system is shown in Fig. 3, and includes four major parts:

(1) Fuzzifier: As external language is expressed in fuzzy concepts, its meaning is transformed into proper language by the fuzzifier created from the membership function in order to be used for the fuzzy operation.

(2) Fuzzy Rule Base: A set of linguistic expressions mainly used to express the control rules, composed of logical operators (if ..., and ..., then ...). These descriptive rules provide four major ways to express the experience, knowledge, and control strategy:

a. Through the knowledge and experience of an expert.

b. According to the control behavior of an operator.

c. According to the characteristics of a control system.

d. Through self-learning.

(3) Fuzzy Inference Engine: The fuzzy inference engine is the core of the fuzzy system. It simulates the thought processes of humans through 


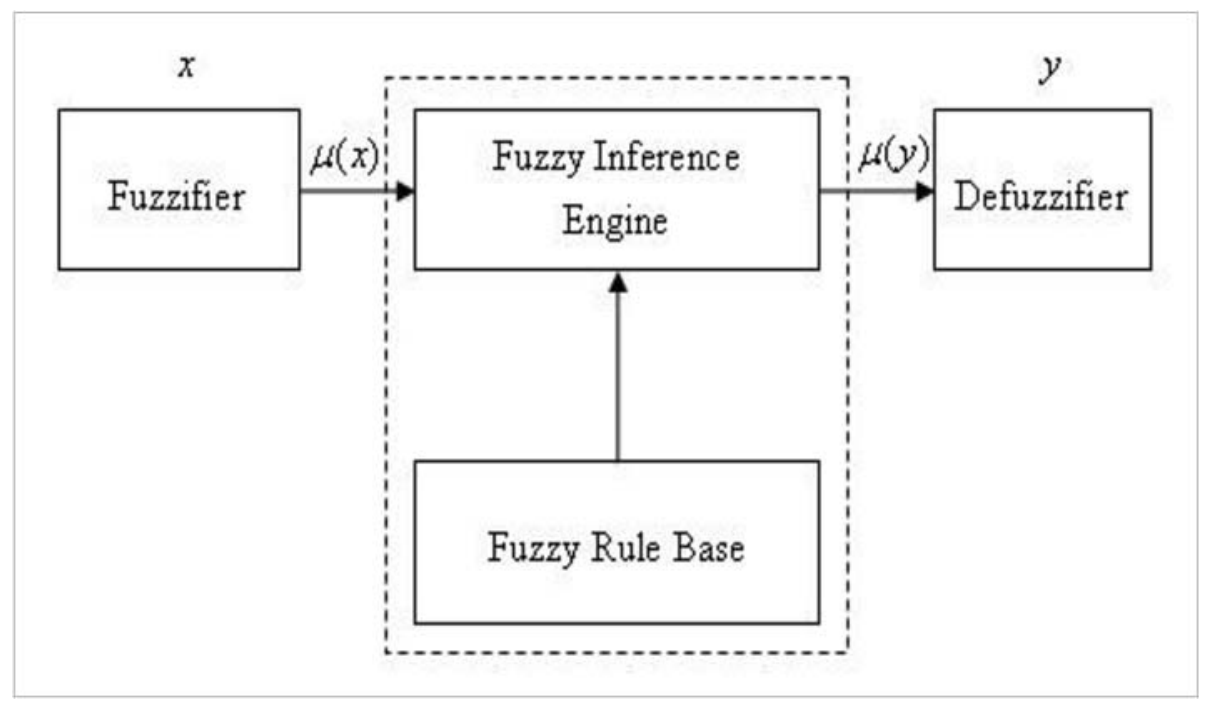

Fig. 3. Fuzzy control system.

an approximation inference for the purpose of problem solving. A fuzzy inference is the inference result generated in accordance with the fuzzy rules. Thus, it is inferred from fuzzy Rule 1, Rule 2, Rule 3, .., Rule n. The process of fuzzy inference can be understood from Fig. 4.

(4) Defuzzifier: The fuzzy inference of the defuzzifier is used to transform fuzzy information into clear information. The main purpose of the defuzzifier is to convert the inference result into a real operation in order to provide a clear output value. There are no definitive defuzzifier methods; the choice depends on the characteristics of the problem. Normally, there are three defuzzifier methods: the Centre of Area (COA), Centre Average Defuzzifier, and Maximum Defuzzifier.

This study utilizes the Centre of Area (COA) for the following calculation (equation (1)):

$y_{0}=\frac{\sum_{i=1}^{m} y_{i} \mu_{c}\left(y_{i}\right)}{\sum_{i=1}^{m} \mu_{c}\left(y_{i}\right)}$

In equation (1), $m$ represents the number of quantization outputs, $y_{i}$ represents the $i$ th value, $\mu_{c}\left(y_{i}\right)$ represents the $y i$ value in fuzzy set $c$, and $y_{0}$ represents the fuzzy output value.

Taking safety into consideration, three linguistic variables are used as inputs: ship length, relative ship speed, and distance. For ship length and relative ship speed, trigonometry can present the linguistic variables. The output is a trigonometric function of linguistic variables for the danger alert index (DAI), as shown in Fig. 5.
Each linguistic variable is defined as follows:

(1) Length (L)

Length has three membership functions: small, medium, and large. It is configured by referencing AIS ship length information. The three functions are defined as follows, and the linguistic set is shown in Table 1.

$\mu_{\text {small }}(x)=\left\{\begin{array}{ll}1 & \text { for } x \leq 130 \\ \frac{190-x}{60} & \text { for } 130 \leq x \leq 190\end{array}\right\}$

$\mu_{\text {medium }}(x)=\left\{\begin{array}{ll}\frac{x-130}{60} & \text { for } 130 \leq x \leq 190 \\ \frac{250-x}{60} & \text { for } 190 \leq x \leq 250\end{array}\right\}$

$\mu_{\text {large }}(x)=\left\{\begin{array}{ll}\frac{x-190}{60} & \text { for } 190 \leq x \leq 250 \\ 1 & \text { for } 250 \leq x\end{array}\right\}$

(2) Relative Speed (RS)

In order to establish the relative speed relationship between two ships, this study utilizes the AIS to receive dynamic and static information from each ship. According to a study of 400 ships in Kinmen Harbor, the average speed of each ship is calculated statistically using the overall average ship speed, which depends on sea state and weather conditions. The study found that the average speed of merchant ships is 10.7 knots, and the standard deviation is 2.2 knots. These linguistic variables are transformed 


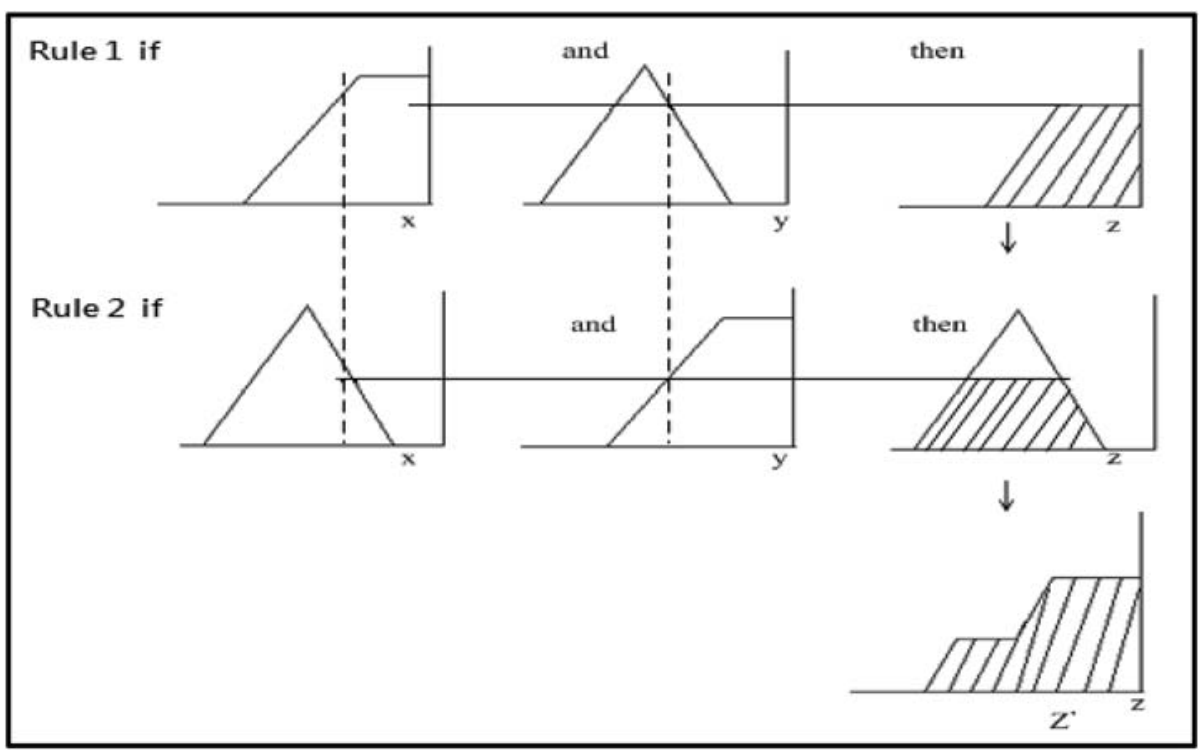

Fig. 4. Schematic diagram of fuzzy inference.

into three ship speed membership functions: slow, middle, and fast. They are defined as follows, and the linguistic set is shown in Table 1.

$$
\mu_{\text {slow }}(y)=\left\{\begin{array}{ll}
1 & \text { for } y \leq 6 \\
\frac{17-y}{11} & \text { for } 6 \leq y \leq 17
\end{array}\right\}
$$

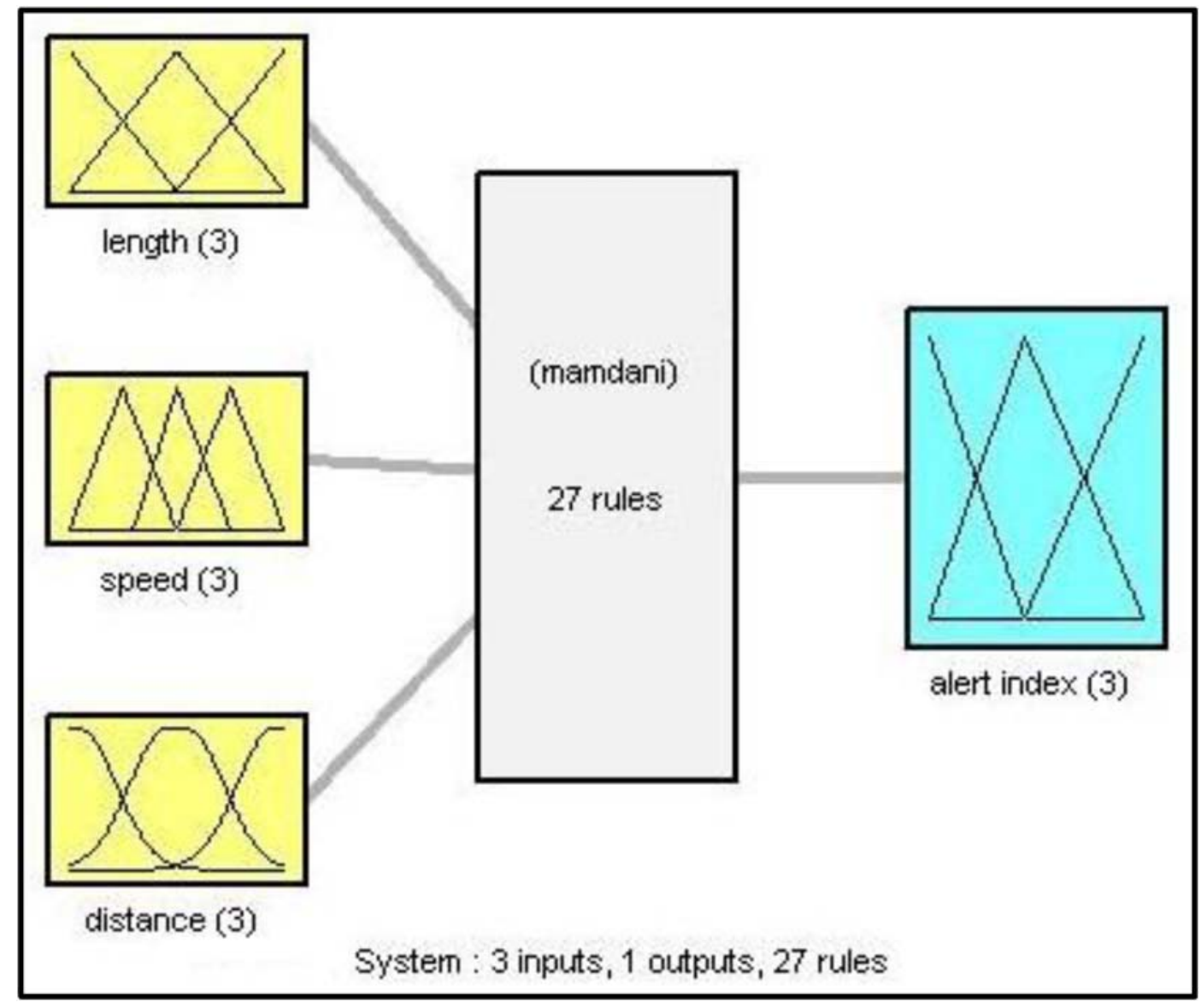

Fig. 5. Fuzzy control system diagrams. 
Table 1. Fuzzy input and output values.

\begin{tabular}{lll}
\hline Input value & & \\
\hline Linguistic variable & Definition & Fuzzy linguistic value \\
\hline Length & Vessel's type and size & $\begin{array}{l}\text { Small (130 Meters) } \\
\text { Medium (190 Meters) } \\
\text { Large (250 Meters) } \\
\text { Slow (6 Knots) } \\
\text { Speed }\end{array}$ \\
& The relative speed between vessel and marine platform & Fiddle (17 Knots) \\
& & Small (1 N.M) \\
Distance & After a ship nears the wind turbines, the valuation of & Medium (3 N.M) \\
& the shortest distance estimates for safely escaping the & Large (5 N.M) \\
\hline Output value & hazardous obstacle facility (rudder use timing) & \\
\hline Linguistic variable & Definition & Fuzzy linguistic value \\
\hline Danger alert index & The degree of danger alert index (1-10) - Green, Amber, or Red. & Small (3-Green light) \\
& Green: continue normal traffic speed. & Medium (5-Amber light, slow flashing) \\
& Amber: Ship operation must be restricted. & Large (10-Red, fast \\
& Red: Stop approaching and change course. & Flashing) \\
\hline
\end{tabular}

$\mu_{\text {medium }}(y)=\left\{\begin{array}{ll}\frac{y-6}{11} & \text { for } 6 \leq y \leq 17 \\ \frac{23-y}{6} & \text { for } 17 \leq y \leq 23\end{array}\right\}$

$\mu_{\text {fast }}(y)=\left\{\begin{array}{ll}\frac{y-17}{6} & \text { for } 17 \leq y \leq 23 \\ 1 & \text { for } 23 \leq y\end{array}\right\}$

\section{(3) Distance (D)}

The study focuses on crash stop distances for different vessels. A survey to investigate the stopping distance required for navigation rudder angle change (for changing course) when a vessel encounters obstacles. Three membership functions for the required distance are defined. According to linguistic variables, they are small, medium, and large. The membership functions are Gaussian functions, and the linguistic set is shown in Table 1.

$$
\begin{aligned}
& \mu_{\text {small }}(z, 1,2,2)=\left\{\exp \left[-\frac{1}{2}\left|\frac{z-1}{2}\right|^{2}\right]\right\} \\
& \mu_{\text {medium }}(z, 3,2,2)=\left\{\exp \left[-\frac{1}{2}\left|\frac{z-3}{2}\right|^{2}\right]\right\} \\
& \mu_{\text {large }}(z, 5,2,2)=\left\{\exp \left[-\frac{1}{2}\left|\frac{z-5}{2}\right|^{2}\right]\right\}
\end{aligned}
$$

\section{(4) Danger Alert Index (DAI)}

The three DAI membership functions are small, medium, and large, with a range of $90^{\circ}$. In fuzzy logic control, the DAI can be treated as an output for danger levels. The present study defines the DAI variable as follows.

$\mu_{\text {small }}(d)=\left\{\begin{array}{ll}1 & \text { for } d \leq 0.5 \\ \frac{4-d}{3.5} & \text { for } 0.5 \leq d \leq 4\end{array}\right\}$
$\mu_{\text {medium }}(d)=\left\{\begin{array}{ll}\frac{d-0.5}{3.5} & \text { for } 0.5 \leq d \leq 4 \\ \frac{8-d}{4} & \text { for } 4 \leq d \leq 8\end{array}\right\}$

$\mu_{\text {large }}(d)=\left\{\begin{array}{ll}\frac{d-4}{4} & \text { for } 4 \leq d \leq 8 \\ 1 & \text { for } 8 \leq d\end{array}\right\}$

The relevant fuzzy linguistic variables, input values, and output values are described in Table 1.

In order to establish a safe collision alert guarding radius model for working platforms, this paper employs the three linguistic variables of length, relative speed, and distance as input values to create a fuzzy alert index. The alert index of the guarding radius is the fuzzy output value. The ship's length and speed data can be obtained from real-time AIS data files, and distance can be calculated using the MGIS. Hence, Length, Speed, and Distance are the inputs, and the Alert Index is the output.

Most membership functions are trigonometric. However, in this study, after several system simulations and assessments by MGIS, and discussed with several Pilots/Captains, according to their long-experienced on navigation. The distance function is not a trigonometric function. Therefore, Gaussian function is used as the input of the 
distance linguistic variable reference value. Different captains have different abilities to judge what distance is hazardous. However, an inexperienced captain may overlook potential dangers and be at greater risk of collision than an experienced captain.

An experienced captain is better at controlling the distance and operating the ship. The peak characteristic of trigonometric functions is not significantly shown and is considered to be closer to a Gaussian distribution. Distance is therefore represented by Gaussian functions as input linguistic variables. In the future, intelligent self-learning modules and faster single-chip calculations will have been designed. The finished plots are shown in Fig. 6.

Based on a professional captain's experience, the linguistic variables of the collision danger index for an offshore work platform or offshore wind turbine may be calculated. The danger warning value is obtained using the FLC method, corresponding to lighting, sound, and AIS radio warning. The fuzzy rules for operation mode are shown in Table 2, and the fuzzy linguistic control intentions are shown in Fig. 7.
Table 2. Fuzzy rules.

\begin{tabular}{lllll}
\hline Linguistic variables & & & \\
\hline Rules & Length (L) & Speed (S) & Distance (D) & DAI (A) \\
\hline Rule 1 & small & small & small & small \\
Rule 2 & small & middle & small & small \\
Rule 3 & small & large & small & medium \\
Rule 4 & small & small & medium & small \\
Rule 5 & small & small & large & small \\
$\ldots$ & $\ldots$ & $\ldots$ & $\ldots$ & $\ldots$ \\
Rule 23 & large & small & large & small \\
Rule 24 & large & middle & medium & medium \\
Rule 25 & large & middle & large & large \\
Rule 26 & large & large & medium & large \\
Rule 27 & large & large & large & large \\
\hline
\end{tabular}

\subsection{Fuzzy collision alert system for OMP}

The system operation cogitation mode and flow chart used in this study is shown in Fig. 8. It is primarily based on the entity model for preventing collisions with platforms at sea. It combines the AIS with MGIS platforms and an FLC to provide its theoretical basis. The length, speed, and distance are used as fuzzy inputs, and the collision alert

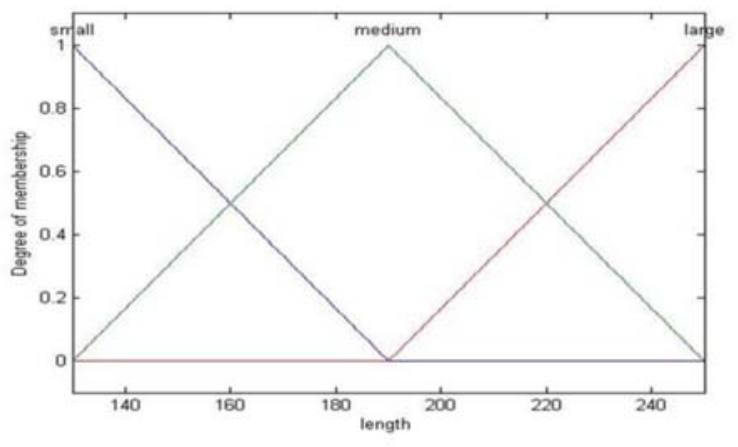

(a)

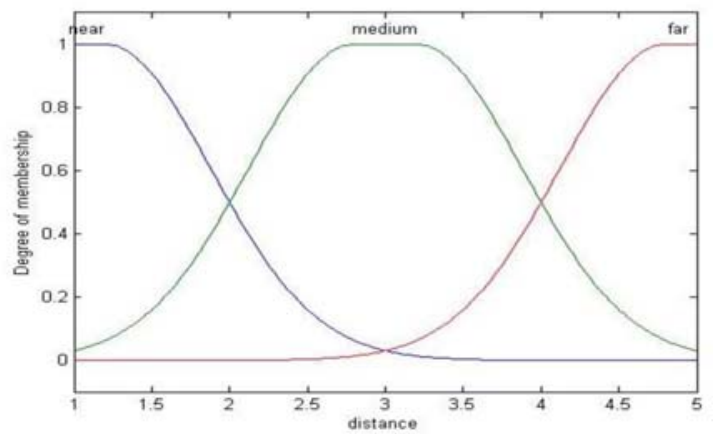

(c)

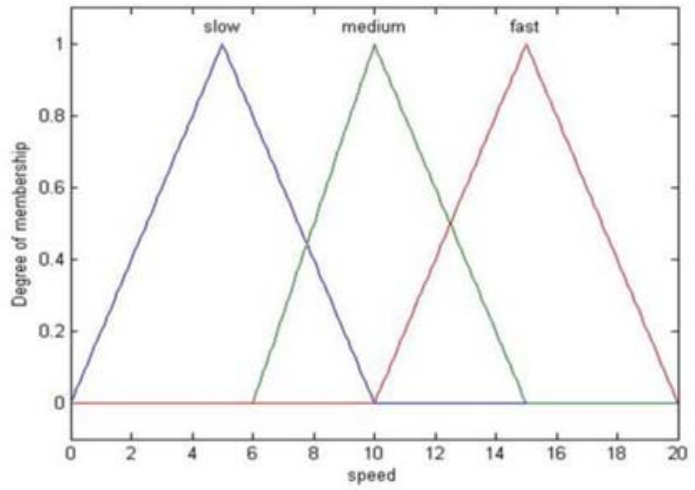

(b)

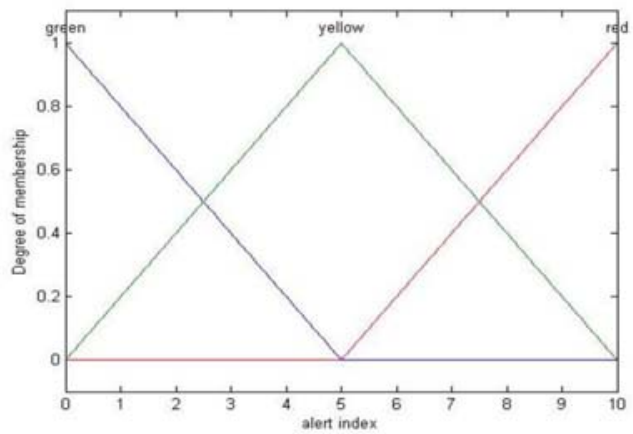

(d)

Fig. 6. Sets of linguistic variables. (a) Ship length: Small, Medium, and Large. (b) Relative speed: Slow, Middle, and Fast. (c) Distance: Small, Medium, and Large. (d) Output alert index: Small, Medium, and Large. 


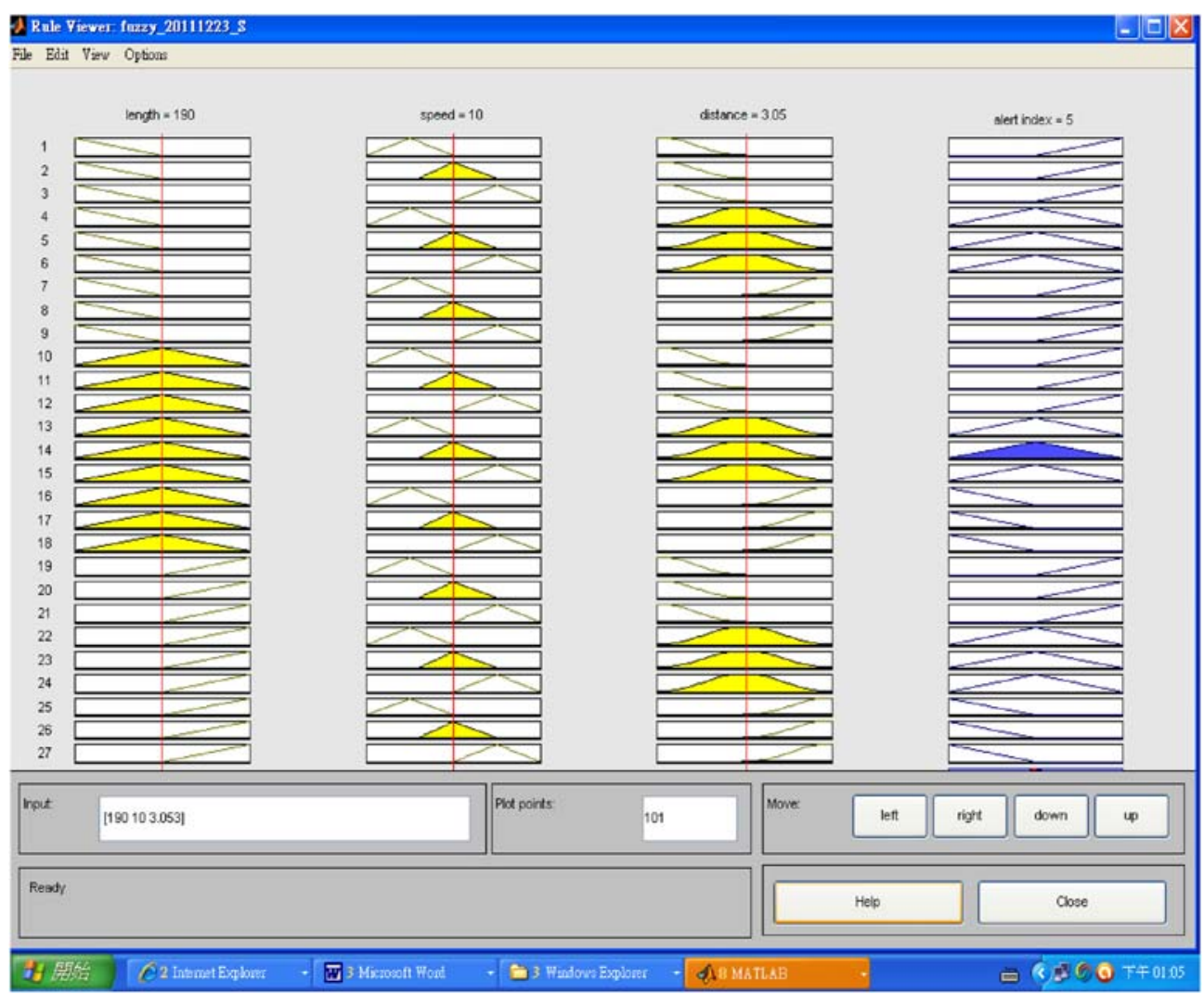

Fig. 7. FLC membership function diagrams.

index is the fuzzy output. The ship system includes AIS collision alerts with a sound and light warning system on the ECDIS and shore VTS. The marine platform itself also provides an audible collision warning system for the vessel is not loaded warning system. The system provides a collision alert for a marine platform with its own alarm and light warning system. But, this paper did not discuss small boat and craft without an installed shipborne AIS.

Because the vessel has been loaded with the AIS, the virtual mode offshore work platform can be used for collision avoidance alerts. Additionally, the VTS and vessel itself provide collision warnings, while the surrounding vessels simultaneously receive immediate relevant collision warnings. The same fuzzy logic control method can also be used for virtual AtoN applications because of the rapid development of radio communication technology and self-organized time division multiple access (SOTDMA) technology. The mobile radio system transmits navigation information that does not actually exist to provide a virtual aid to navigation and is set on a particular platform using a fuzzy control method to calculate the degree of danger.
After solving these proposed warning indicators for different degrees of risk of collision, this system will broadcast the alert information by AIS without setting an entity aids facility. The system will automatically warn all nearby vessels' navigators, VTS duty officers, and offshore work platform operators. Hence, it can identify the degree of danger to provide collision alerts for any weather or ambiguous conditions in order to enhance and highlight navigational safety. This will be the newest trend in the buildup of necessary offshore collision alert aids.

\section{Simulation and analysis}

This was a cooperative study with a communications corporation to develop the AIS and maritime GIS tablet shown in Fig. 9. After several tests and modifications, the system was completed with real field measurements and system integration in Taiwan over a period of three years. Radio transmission coverage testing by the AIS and a successful display of the vessels' tracking are shown in Fig. 10. This AIS system can provide immediate aids to navigation, provide ships with hazardous obstacle beacon locations for marine platforms, and provide navigation information facilities. An example of a 


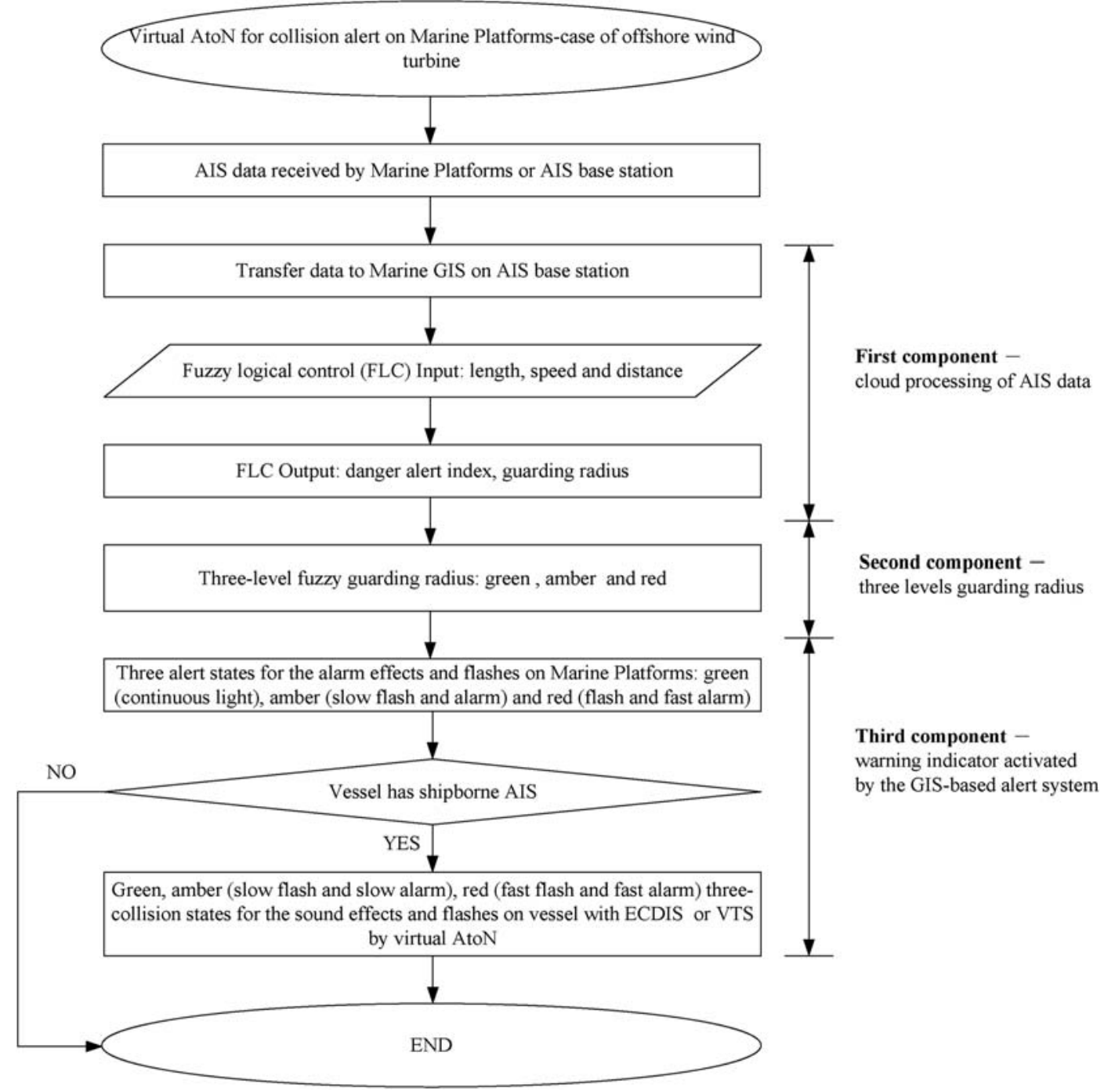

Fig. 8. Flow chart of GIS-based alert system for offshore marine platform.
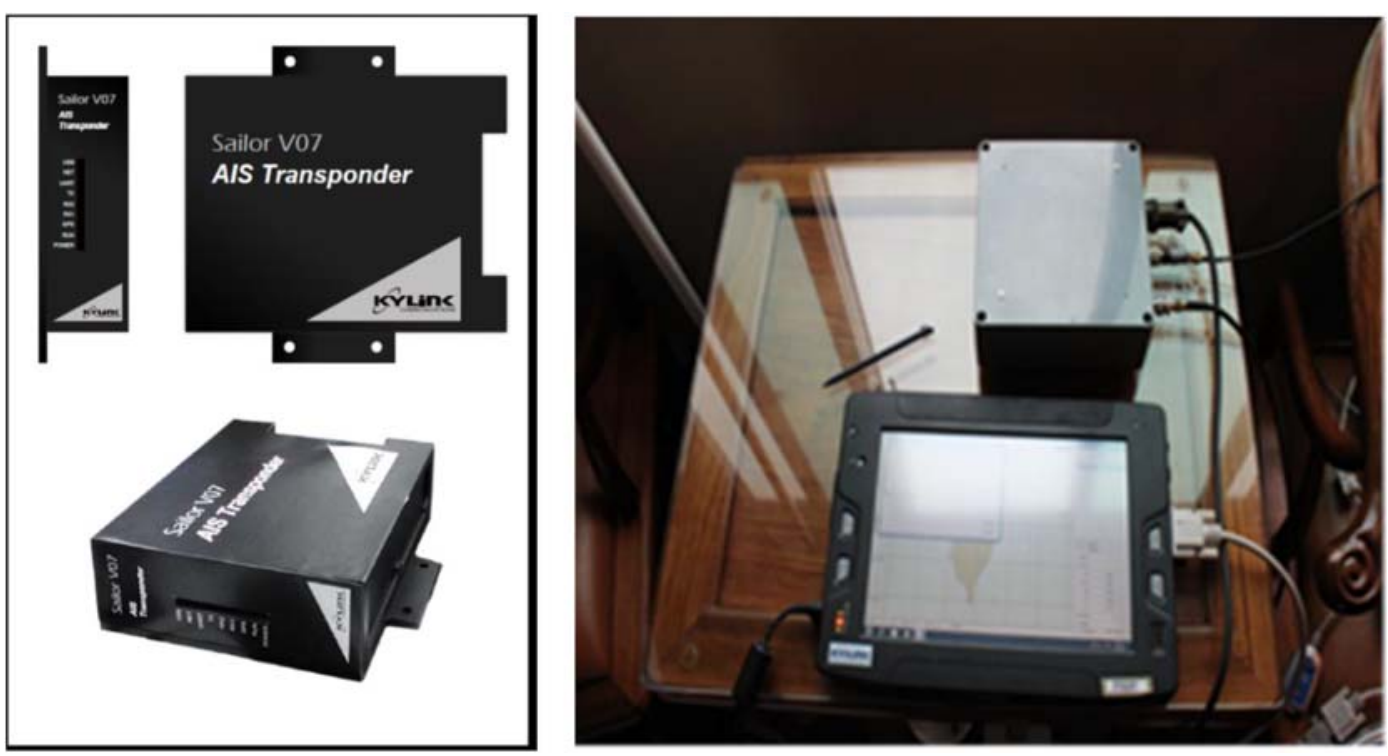

Fig. 9. Research developed AIS and maritime GIS tablet. 


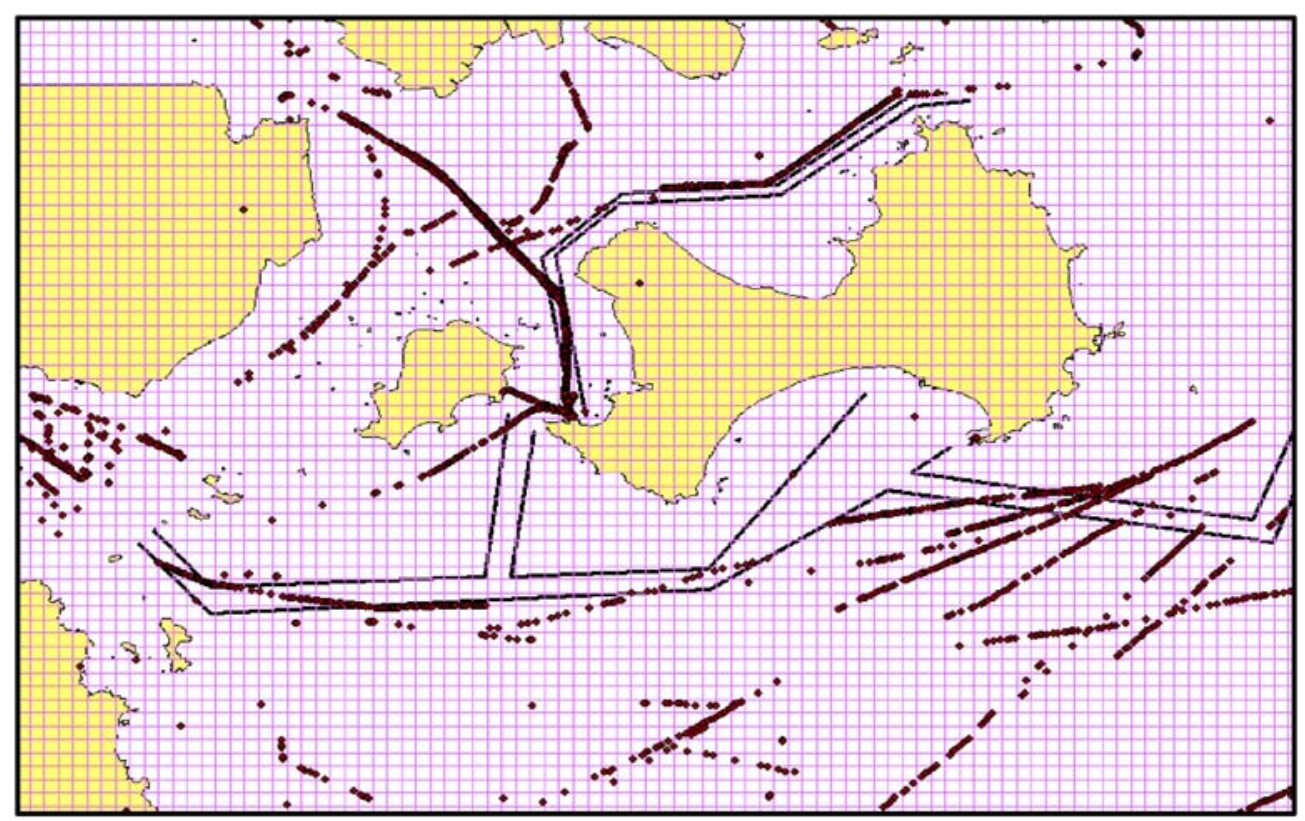

Fig. 10. Radio transmission coverage and ship tracking test by AIS.

fuzzy alert schematic chart is shown in Fig. 11. Offshore wind turbines are located at the black circles, and the "+ " symbol represents the AIS base station. The collision alert device (warning light and alarm) equipped on the work platform is controlled by the MGIS/VTS shore station via radio communication. If the ship length is $190 \mathrm{~m}$, the ship speed is 10 knots, and the distance to OMP is 3.06 nautical miles, then the cloud processing output DAI is 5, and the amber light shows on the OMP and MGIS. There is also a slow flash to alert the navigator to take action for collision avoidance. If the ship is approaching the work platform, and the navigator has made no response to this warning as the ship approaches the red line (the cloud calculated distance to reach a DAI of 7 or higher), a higher alert level will be activated. Once the distance is equal to or less than 1 nautical mile, the system will activate a flashing red light and treble alarm to warn the navigator. Thus, the navigator, once alerted to the warning signal, will immediately change course to adjust the ship's heading for collision avoidance. The flashing light and alarm will continue until the ship returns to the green alert band.

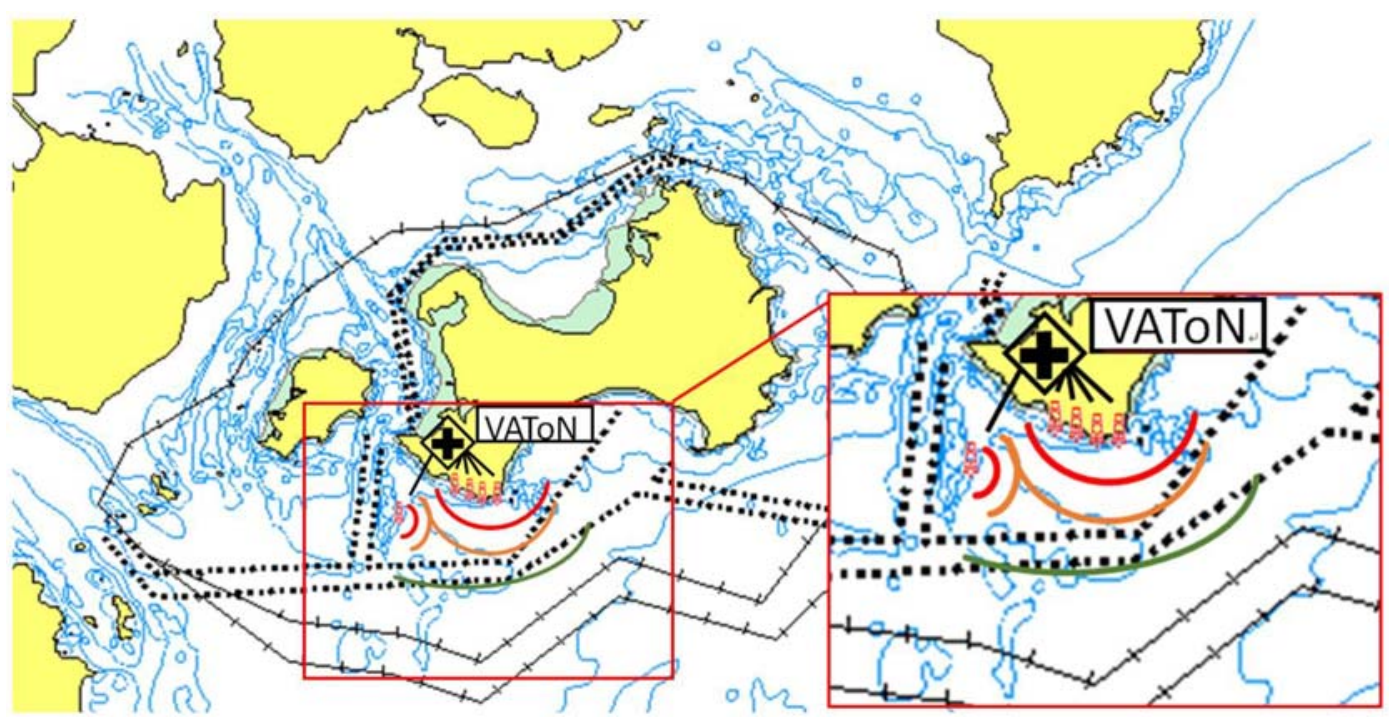

Fig. 11. Schematic diagram of risk value and warning indicator for offshore wind turbines on MGIS. 
This system has been developed for VTS, Officer on watch (OOW) onboard, and working platforms, which equip each kind of AIS at the base station, on ships, and in a virtual station. The Marine GIS is now used to monitor marine navigation safety and environmental protection. Therefore, these mechanisms can be used as a novel function for an advanced ECDIS on board after approval by IALA and IMO.

\section{Conclusion}

This paper proposed a novel fuzzy collision alert system using a MGIS for the navigation safety of marine platforms. As the FGR/SD will be larger with poor weather conditions and low visibility at sea, therefore, the three-level fuzzy guarding radius: green, amber and red are changeable. The system uses single-chip implanted AIS Class B technology and FMGIS to effectively initiate an alert for all ships' navigators. With this strategy, VTS duty operators and offshore work platform workers will be warned of dangers and urgent situations before a collision occur. The application of the system can actively protect wind farms from vessels collision with only several third GIS components (activate alarm devices). This system has been implemented in the offshore west coast wind farms in the Taiwan sea area to reduce vessel collisions with offshore marine platforms.

In the future, the system to process cloud AIS data can be combined with ship simulators. Besides offering the alert of flash and alarm, the approaching ship will obtain the advice of rudder angle changes from the system to avoid collision with OMPs.

\section{References}

[1] Bela A, Le Sourne H, Buldgen L, Rigo P. Numerical crashworthiness analysis of an offshore wind turbine monopile impacted by a ship. Analysis and design of marine structures. 2015. p. 661-9.

[2] Bela A, Pire T, Buldgen L, Rigo P. Ship collision on offshore wind turbines. Pianc Yearbook 2016;2015:189.
[3] Buckley JJ. Fuzzy hierarchical analysis. Fuzzy Set Syst 1985, 17:233-47.

[4] Fenton A. Fishermen rescued from sinking boat after crashing into Barrow wind turbine while on auto-pilot. 2017. Retrieved from: http://www.nwemail.co.uk/news/barrow/ Fishermen-rescued-from-sinking-boat-after-crashing-intoBarrow-wind-turbine-while-on-auto-pilot-593ebaef-26b64e6a-8582-4712094cca5e-ds.

[5] Goodwin EM. A statistical study of ship domains. J Navig $1975 ; 28: 328-44$.

[6] Hsu H-Z, Witt NA, Hooper JB, McDermott AP. The AISassisted collision avoidance. J Navig 2009;62:657-70.

[7] IALA Recommendation A-126 - the use of the automatic identification systems (AIS) in marine aids to navigation services. 2011.

[8] Kao S-L, Lee K-T, Chang K-Y, Ko M-D. A fuzzy logic method for collision avoidance in vessel traffic service. J Navig 2007; 60:17-31.

[9] Koldenhof Y, Van der Tak C, Glansdorp C. Risk Awareness; a model to calculate the risk of a ship dynamically. 2009. p. 112-9.

[10] Kramer B. Assessing the consequences of sea-vessel collisions with offshore wind farms: Part I. 2014. Retrieved from: http://www.renewableenergyfocus.com/view/38450/ assessing-the-consequences-of-sea-vessel-collisions-withoffshore-wind-farms-part-i/.

[11] Lü G, Batty M, Strobl J, Lin H, Zhu A-X, Chen M. Reflections and speculations on the progress in Geographic Information Systems (GIS): a geographic perspective. Int J Geogr Inf Sci 2019;33:346-67.

[12] Li R, Fan J, Jiang J, Wu H. Spatiotemporal correlation in WebGIS group-user intensive access patterns. Int J Geogr Inf Sci 2017;31:36-55.

[13] Mehdi RA, Baldauf M, Deeb H. A dynamic risk assessment method to address safety of navigation concerns around offshore renewable energy installations. Proc IME M J Eng Marit Environ 2019;234(1):231-44.

[14] Mou JM, Van Der Tak C, Ligteringen H. Study on collision avoidance in busy waterways by using AIS data. Ocean Eng 2010;37:483-90.

[15] Presencia CE, Shafiee M. Risk analysis of maintenance ship collisions with offshore wind turbines. Int J Sustain Energy 2017:1-21.

[16] Pyman M, Austin J, Lyon P. Ship/platform collision risk in the UK sector. 1983.

[17] Szlapczynski R, Szlapczynska J. An analysis of domain-based ship collision risk parameters. Ocean Eng 2016;126:47-56.

[18] Tsou M-C. Online analysis process on Automatic Identification System data warehouse for application in vessel traffic service. Proc IME M J Eng Marit Environ 2016;230: 199-215.

[19] Wright RG, Baldauf M. Virtual electronic aids to navigation for remote and ecologically sensitive regions. J Navig 2017; 70:225-41.

[20] Zadeh LA. Fuzzy sets. Inf Contr 1965;8:338-53. 